\title{
Approximate Series Solutions for Nonlinear Free Vibration of Suspended Cables
}

\author{
Yaobing Zhao, ${ }^{1}$ Ceshi Sun, ${ }^{1}$ Zhiqian Wang, ${ }^{2}$ and Lianhua Wang ${ }^{1}$ \\ ${ }^{1}$ College of Civil Engineering, Hunan University, Changsha, Hunan 410082, China \\ ${ }^{2}$ College of Mechanical and Vehicle Engineering, Hunan University, Changsha, Hunan 410082, China \\ Correspondence should be addressed to Yaobing Zhao; ybzhao@hnu.edu.cn
}

Received 16 October 2013; Accepted 24 February 2014; Published 20 March 2014

Academic Editor: Didier Rémond

Copyright (c) 2014 Yaobing Zhao et al. This is an open access article distributed under the Creative Commons Attribution License, which permits unrestricted use, distribution, and reproduction in any medium, provided the original work is properly cited.

\begin{abstract}
This paper presents approximate series solutions for nonlinear free vibration of suspended cables via the Lindstedt-Poincare method and homotopy analysis method, respectively. Firstly, taking into account the geometric nonlinearity of the suspended cable as well as the quasi-static assumption, a mathematical model is presented. Secondly, two analytical methods are introduced to obtain the approximate series solutions in the case of nonlinear free vibration. Moreover, small and large sag-to-span ratios and initial conditions are chosen to study the nonlinear dynamic responses by these two analytical methods. The numerical results indicate that frequency amplitude relationships obtained with different analytical approaches exhibit some quantitative and qualitative differences in the cases of motions, mode shapes, and particular sag-to-span ratios. Finally, a detailed comparison of the differences in the displacement fields and cable axial total tensions is made.
\end{abstract}

\section{Introduction}

As a basic and significant structural element, the suspended cable has been widely applied in many mechanical systems and engineering fields $[1,2]$, such as civil, ocean, and aerospace engineering. Generally speaking, the nonlinear dynamics of the suspended cable is very complicated and attracts more and more attention in recent years, for example, referring to the literature reviews by Rega $[3,4]$.

The suspended cable is a typical weakly nonlinear continuous system which contains the quadratic and cubic nonlinearity terms, and the nonlinear free vibration of the system has been studied through many analytical methods in the previous researches. Recently, Hagedorn and Schäfer [5] investigated the nonlinear free vibrations of suspended cables in the case of small sag via the Lindstedt method. Luongo et al. [6, 7] applied multiple scales method and Lindstedt-Poincare method to study the nonlinear planar free vibrations of an elastic cable, respectively. Rega et al. [8] examined the nonlinear phenomenon in a large range of the cable sag-to-span ratios by the numerical investigation. Benedettini et al. [9] applied an order-three perturbation expansion to obtain the solutions of the free nonplanar coupled equations. Srinil et al. [10] presented a model to analyze large amplitude free vibrations of the suspended cable in three dimensions. In these studies, the perturbation method is the most significant analytical way to study the nonlinear vibration of the suspended cable. However, it is noted that the perturbation method should be based on the small parameter assumption. Because of the limitations in the perturbation method, its application is restricted, more or less. On the other hand, due to the limitations of the perturbation method, some nonperturbation methods are introduced to study the nonlinear free vibrations of suspended cables in three dimensions, for instance, the harmonic balance method [11]. Nevertheless, the nonperturbation method could not provide us with a way to guarantee the convergence of the series solutions.

Hence, in order to overcome the limitations in both the perturbation method and the nonperturbation method, Liao [12] proposed a general analytical method which is the homotopy analysis method. On the one hand, it has been successfully applied in many engineering fields with its further development in recent years [13]. For example, 
Hoseini et al. [14] obtained accurate analytical results for the nonlinear free vibration of a conservative oscillator with cubic nonlinearity by using homotopy analysis method. Pirbodaghi et al. [15] investigated the nonlinear vibration behavior of Euler-Bernoulli beams subjected to axial load via homotopy analysis method. Kargarnovin et al. [16] applied homotopy analysis method to analyze the nonlinear free vibrations of the simple end beams. Qian et al. $[17,18]$ employed homotopy analysis method to obtain approximate solutions for an electrostatically actuated microbeam and an elastically restrained beam with a lumped mass, respectively. Wu et al. [19] contributed to the research of the nonlinear thickness-shear vibrations of a finite crystal plate with homotopy analysis method.

On the other hand, there are some comparisons between the homotopy analysis method and perturbation method. Specifically, Yuan and Li [20] found that the solutions of homotopy analysis method agree well with the results of the modified Linstedt-Poincare method and incremental harmonic balance method for the primary resonance of multiple degree-of-freedom dynamic system with strong nonlinearity. Comparisons were made between Adomian's decomposition method and homotopy analysis method by Tan and Abbasbandy [21]. You and $\mathrm{Xu}$ [22] studied the analytical approximations for the periodic motion of the duffing system with delayed feedback via the homotopy analysis method and multiple scales method and pointed out that the results obtained with the multiple scales method were less accurate.

To the best knowledge of the authors, no specific study has addressed a comparison of homotopy analysis method and other perturbation method in the case of nonlinear free vibrations of the suspended cable. However, the homotopy analysis method is different from those presented in the papers published before on the same problem, so this research will focus on the comparison of these methods. The paper consists of four sections: firstly, the nonlinear free vibration equations of motion are derived by applying the Homilton's principle and quasi-static assumption, and then the multimode expansion of the displacement is introduced to obtain a discrete cable model. Secondly, the approximate series solutions obtained with Lindstedt-Poincare method and homotopy analysis method are constructed, respectively. Moreover, in the section of the numerical analysis, time histories, frequency amplitude curves, displacement fields, and axial tension forces of suspended cables are compared and illustrated. Finally, some conclusions are made at the end of the paper.

\section{Mathematical Model}

Figure 1 shows the coordinate system and two configurations of the suspended cable (the initial deformed configuration of static equilibrium under its own weight and the dynamic configuration occupied during the vibration). As described in this figure, the left support $O$ is the origin of the coordinate, the direction $O B$ is taken as the $x$-coordinate, and the direction perpendicular to $O B$ is the $y$-direction of which the descending direction is taken as positive. The displacements

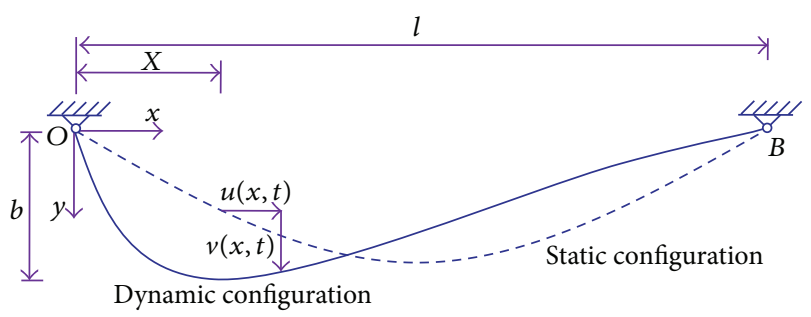

Figure 1: Two different configurations of the suspended cable.

of the point are described by $u(x, t)$ and $v(x, t)$ along the longitudinal $x$ and vertical $y$ directions, respectively.

2.1. Equations of Motion. By applying the Hamilton's principle and the quasi-static stretching assumption, we could express the nonlinear partial differential equation of motion without considering the bending, torsional, and shear rigidities as [3]

$$
m \ddot{v}-H v^{\prime \prime}-\frac{E A}{l}\left(y^{\prime \prime}+v^{\prime \prime}\right) \int_{0}^{l}\left(y^{\prime} v^{\prime}+\frac{1}{2} v^{\prime 2}\right) \mathrm{d} x=0,
$$

where $m$ is the mass per unit length, $A$ is the uniform crosssectional area, $E$ is the modulus of elasticity, $H$ is the horizontal component of the tension $\left(H=m g^{2} / 8 b, H / E A \ll 1\right), l$ is the span, $b$ is the sag at the midspan, and $g$ is the acceleration due to gravity.

The corresponding boundary conditions are written as

$$
v(x, t)=0 \quad \text { at } x=0, x=l .
$$

In this study, because the sag-to-span ratio is sufficiently small $(f=b / l<1 / 8)$, the static equilibrium configuration is described well through a parabola:

$$
y(x)=4 f\left[\frac{x}{l}-\left(\frac{x}{l}\right)^{2}\right] .
$$

2.2. Condensed Model. In order to make the subsequent section more general, the following nondimensional quantities are adopted [23]:

$$
\begin{gathered}
v^{*}=\frac{v}{l}, \quad x^{*}=\frac{x}{l}, \quad y^{*}=\frac{y}{l}, \\
t^{*}=\sqrt{\frac{g}{8 f}} t, \quad \alpha=\frac{E A}{H},
\end{gathered}
$$

where the nondimensionalization with respect to the span which affects the range of nondimensionalized response amplitude is adopted.

As a result, (1) can be written as follows:

$$
y(x)=4 f\left[\frac{x}{l}-\left(\frac{x}{l}\right)^{2}\right]
$$

where the asterisks in (5) are omitted for simplicity, $y(x)=$ $4 f x(1-x)$ is the nondimensional initial parabolic shape, and the boundary conditions associated with (5) are given as

$$
v(x, t)=0 \quad \text { at } x=0, x=1 .
$$


2.3. Mode Shapes and Frequencies. Eliminating the nonlinear terms, the mode shapes, and frequencies can be ascertained by solving linearized equation of motion. Therefore, the inplane $n$th $(n=$ odd) symmetric mode shapes are derived by

$$
\begin{array}{r}
\varphi_{n}(x)=\xi_{n}\left[1-\cos \left(\omega_{n} x\right)-\tan \left(\frac{\omega_{n}}{2}\right) \sin \left(\omega_{n} x\right)\right], \\
(n=1,3,5, \ldots),
\end{array}
$$

where the coefficients $\xi_{n}$ are derived by the normalization conditions of modes and the mode frequencies $\omega_{n}$ in (7) are obtained by solving the following transcendental equation:

$$
\tan \left(\frac{\omega_{n}}{2}\right)=\frac{\omega_{n}}{2}-\frac{1}{2 \lambda^{2}} \omega_{n}^{3}, \quad(n=1,3,5, \ldots),
$$

where $\lambda^{2}=E A / m g L(8 b / L)^{3}$ is the Irvine parameter which is an important factor in the geometrical and mechanical properties of the suspended cable.

On the other hand, the $n$th $(n=$ even $)$ in-plane antisymmetric mode shapes and relative frequencies are

$$
\varphi_{n}(x)=\sqrt{2} \sin (n \pi x), \quad \omega_{n}=n \pi, \quad(n=2,4,6, \ldots) .
$$

2.4. Discrete Model. Assuming that the suspended cable is a multi-degree-of-freedom (MDOF) dynamic system, which is composed of symmetric and antisymmetric modes with respect to the midspan, the Galerkin method is employed to simplify the nonlinear oscillation equation of motion. Considering the boundary conditions, the solutions of (5) are expanded into the following expression:

$$
v(x, t)=\sum_{n=1}^{N} q_{n}(t) \varphi_{n}(x),
$$

where $N$ is the number of modes used in the approximation $(N=1,2, \ldots \infty), q_{n}(t)$ is an unknown function of time which is a generalized coordinate of the system response, and $\varphi_{n}(x)$ is a space coordinate function satisfying the associated linear problem.

Therefore, a set of nonlinear ordinary differential equations are yielded by substituting (10) into (5):

$$
\ddot{q}_{n}+\omega_{n}^{2} q_{n}+\sum_{i=1}^{N} \sum_{j=1}^{N} \Gamma_{n i j} q_{i} q_{j}+\sum_{i=1}^{N} \sum_{j=1}^{N} \sum_{h=1}^{N} \Lambda_{n i j h} q_{i} q_{j} q_{h}=0,
$$

where the dots denote derivatives with respect to $t$ and the expressions of the coefficients of the quadratic and cubic terms in (11) are as follows:

$$
\begin{aligned}
\Lambda_{n i j h}= & -\frac{1}{2} \alpha \int_{0}^{1}\left[\varphi_{j}^{\prime \prime}(x) \int_{0}^{1} \varphi_{i}^{\prime}(x) \varphi_{h}^{\prime}(x) \mathrm{d} x\right] \varphi_{n}(x) \mathrm{d} x ; \\
\Gamma_{n i j}= & -\alpha \int_{0}^{1}\left[\varphi_{j}^{\prime \prime}(x) \int_{0}^{1} y^{\prime}(x) \varphi_{i}^{\prime}(x) \mathrm{d} x\right] \varphi_{n}(x) \mathrm{d} x \\
& -\frac{1}{2} \alpha \int_{0}^{1}\left[y^{\prime \prime}(x) \int_{0}^{1} \varphi_{i}^{\prime}(x) \varphi_{i}^{\prime}(x) \mathrm{d} x\right] \varphi_{n}(x) \mathrm{d} x .
\end{aligned}
$$

\section{Methods of Solution}

This section begins with the approximate series solutions for the nonlinear free vibrations obtained with the Lindstedt-Poincare method, followed by the homotopy analysis method. For the sake of simplicity, only single-mode model (symmetric mode or antisymmetric mode) is considered.

3.1. Lindstedt-Poincare Method. Firstly, a new independent variable is introduced, which is

$$
\tilde{t}=\omega_{n} t
$$

where $\omega_{n}$ is the linear frequency of the suspended cable. Therefore, (11) is transformed into

$$
\ddot{q}_{n}+q_{n}+\frac{\Gamma_{n n n}}{\omega_{n}^{2}} q_{n}^{2}+\frac{\Lambda_{n n n n}}{\omega_{n}^{2}} q_{n}^{3}=0
$$

where the coefficient of the linear term is equal to unity since the time is nondimensionalized with respect to the linear vibration frequency.

By assuming an expansion for $q_{n}=\varepsilon \widetilde{q}_{n}$ (where $\varepsilon$ is a small finite parameter) and omitting the tilde, we obtain

$$
\ddot{q}_{n}+q_{n}+\varepsilon \frac{\Gamma_{n n n}}{\omega_{n}^{2}} q_{n}^{2}+\varepsilon^{2} \frac{\Lambda_{n n n n}}{\omega_{n}^{2}} q_{n}^{3}=0 .
$$

Following the method of Lindstedt-Poincare [7], we seek the 4 th order approximate solution to (15) by letting

$$
q_{n}(\tilde{t} ; \varepsilon)=\sum_{m=0}^{4} \varepsilon^{m} q_{n m}(t)
$$

and a strained time coordinate $\tau$ is introduced:

$$
\tau=\left(1+\sum_{m=1}^{4} \varepsilon^{m} \alpha_{m}\right) \tilde{t}
$$

Then, we could obtain the relation between the nonlinear frequency $\Omega_{n}$ and the linear one $\omega_{n}$ as follows:

$$
\frac{\Omega_{n}-\omega_{n}}{\omega_{n}}=\sum_{m=1}^{4} \varepsilon^{m} \alpha_{m}
$$

Substituting (16) and (17) into (15) and equating the coefficients $\varepsilon^{m}$ on both sides, the nonlinear ordinary equations are reduced to a set of linearized equations. Then, the polar form is introduced and the secular terms are set to zero. Hence, the series solutions of $q_{n m}$ and $\alpha_{m}$ are obtained, based on the fact 
that the 4th order series solutions of the frequency amplitude relationship and displacement are as follows [7]:

$$
\begin{aligned}
\Omega_{n}=\omega_{n}[1+ & \left(\frac{3}{8} \frac{\Lambda_{n n n n}}{\omega_{n}^{2}}-\frac{5}{12} \frac{\Gamma_{n n n}^{2}}{\omega_{n}^{4}}\right) a^{2} \\
& +\left(-\frac{15}{256} \frac{\Lambda_{n n n n}^{2}}{\omega_{n}^{4}}-\frac{485}{1728} \frac{\Gamma_{n n n}^{4}}{\omega_{n}^{8}}\right. \\
& \left.\left.+\frac{173}{192} \frac{\Lambda_{n n n n}}{\omega_{n}^{2}} \frac{\Gamma_{n n n}^{2}}{\omega_{n}^{4}}\right) a^{4}\right], \\
q_{n}(t)=a \cos (\Omega & +\beta)+a^{2}\left[\frac{\Gamma_{n n n}}{6 \omega_{n}^{2}} \cos (2 \Omega t+2 \beta)-\frac{\Gamma_{n n n}}{2 \omega_{n}^{2}}\right] \\
+a^{3}( & \left.\frac{\Gamma_{n n n}^{2}}{48 \omega_{n}^{4}}+\frac{\Lambda_{n n n n}}{32 \omega_{n}^{2}}\right) \cos (3 \Omega t+3 \beta) \\
+a^{4}[ & \left(-\frac{31}{96} \frac{\Gamma_{n n n}}{\omega_{n}^{2}} \frac{\Lambda_{n n n n}}{\omega_{n}^{2}}+\frac{59}{432} \frac{\Gamma_{n n n}^{3}}{\omega_{n}^{6}}\right) \\
& \times \cos (2 \Omega t+2 \beta) \\
& +\left(\frac{5}{8} \frac{\Gamma_{n n n}}{\omega_{n}^{2}} \frac{\Lambda_{n n n}}{\omega_{n}^{2}}-\frac{19}{72} \frac{\Gamma_{n n n}^{3}}{\omega_{n}^{6}}\right) \\
& +\left(\frac{1}{432} \frac{\Gamma_{n n n}^{3}}{\omega_{n}^{6}}+\frac{1}{96} \frac{\Gamma_{n n n}}{\omega_{n}^{2}} \frac{\Lambda_{n n n n}}{\omega_{n}^{2}}\right) \\
& \times \cos (4 \Omega t+4 \beta)], \\
& (49)
\end{aligned}
$$

where $a$ is the actual nondimensional response amplitude, $\beta$ is the phase of the oscillation, and $t$ is the actual time scale. Moreover, there is a drift term due to the quadratic nonlinearity in (20), indicating that the equilibrium position is not at $q=0$.

3.2. Homotopy Analysis Method. In the following, the nonlinear free response of the suspended cable is explored by homotopy analysis method which transforms a nonlinear problem into an infinite number of linear problems with an embedding parameter $q$ that typically varies from 0 to 1 .

Introducing a new time scale $\tau=\Omega_{n} t\left(\Omega_{n}\right.$ is the nonlinear vibration frequency) and taking into account the quadratic nonlinear term, we suppose that

$$
q_{n}(t)=u_{n}(\tau)+\delta_{n}
$$

In (11), the initial conditions are assumed to be

$$
q_{n}(0)=b_{n 0}+\delta_{n}, \quad \dot{q}_{n}(0)=0,
$$

where $b_{n 0}$ is the initial condition and $\delta_{n 0}$ is the nonzero equilibrium position term due to the quadratic nonlinearity.
Under the new time scale transformation, the new form of (11) is

$$
\begin{aligned}
& \Omega_{n}^{2} \ddot{u}_{n}(\tau)+\omega_{n}^{2}\left[u_{n}(\tau)+\delta_{n}\right]+\Gamma_{n n n}\left[u_{n}(\tau)+\delta_{n}\right]^{2} \\
& +\Delta_{n n n n}\left[u_{n}(\tau)+\delta_{n}\right]^{3}=0,
\end{aligned}
$$

where

$$
\ddot{u}_{n}(\tau)=\frac{\mathrm{d}^{2} u_{n}(\tau)}{\mathrm{d} \tau^{2}} .
$$

Therefore, the corresponding initial conditions are

$$
u_{n}(0)=b_{n 0}, \quad \dot{u}_{n}(0)=0 .
$$

Given the fact that the free oscillations of a conservative system could be expressed by a series of periodic functions which satisfy the initial conditions:

$$
\{\cos (k \tau) \mid k=1,2,3, \ldots\},
$$

the displacement solution of (23) can be expressed by

$$
u_{n}(\tau)=\sum_{k=1}^{+\infty} C_{n k} \cos (k \tau) .
$$

Considering the rule of solution expression and initial conditions in (25), the initial guess of $u_{n}(\tau)$ is chosen as

$$
u_{n 0}(\tau)=b_{n 0} \cos \tau \text {. }
$$

To construct the homotopy function, one may define the linear auxiliary operator as

$$
\mathscr{L}\left[\Phi_{n}(\tau ; q)\right]=\omega_{n 0}^{2}\left[\frac{\partial^{2} \Phi_{n}(\tau ; q)}{\partial \tau^{2}}+\Phi_{n}(\tau ; q)\right],
$$

which has the property

$$
\mathscr{L}\left(C_{1} \sin \tau+C_{2} \cos \tau\right)=0,
$$

for any integration constants $C_{1}$ and $C_{2}$.

According to (23), we could define the nonlinear operator as

$$
\begin{aligned}
\mathcal{N} & {\left[\Phi_{n}(\tau ; q), \Delta_{n}(q), \Psi_{n}(q)\right] } \\
= & \Psi_{n}^{2}(q)\left[\frac{\partial^{2} \Phi_{n}(\tau ; q)}{\partial \tau^{2}}\right]+\omega_{n}^{2}\left[\Phi_{n}(\tau ; q)+\Delta_{n}(q)\right] \\
& +\Gamma_{n n n}\left[\Phi_{n}(\tau ; q)+\Delta_{n}(q)\right]^{2}+\Lambda_{n n n n}\left[\Phi_{n}(\tau ; q)+\Delta_{n}(q)\right]^{3},
\end{aligned}
$$

where the unknown function $\Phi_{n}(\tau ; q)$ is a mapping of $u_{n}(\tau)$ and the unknown functions $\Psi_{n}(q)$ and $\Delta_{n}(q)$ are some kinds of mapping of the unknown nonlinear frequency $\Omega_{n}$ and the equilibrium position $\delta_{n}$, respectively. In accordance with the homotopy analysis method, we construct the zeroth order deformation equation as

$$
\begin{aligned}
& (1-q) \mathscr{L}\left[\Phi_{n}(\tau ; q)-u_{n 0}(\tau)\right] \\
& \quad=q \hbar H(\tau) \mathscr{N}\left[\Phi_{n}(\tau ; q), \Delta_{n}(q), \Psi_{n}(q)\right],
\end{aligned}
$$


subjected to the initial conditions

$$
\Phi_{n}(0 ; q)=b_{n 0},\left.\quad \frac{\partial \Phi_{n}(\tau ; q)}{\partial \tau}\right|_{\tau=0}=0,
$$

where $q \in[0,1]$ is an embedding parameter, $\hbar \neq 0$ is an auxiliary convergence control parameter, $H(\tau) \neq 0$ is an auxiliary function, and $\mathscr{L}(\mathcal{N})$ is an auxiliary linear (nonlinear) operator.

For the sake of simplicity, we choose

$$
H(\tau)=1 .
$$

Therefore, with the increase of the embedding parameter $q$ from 0 to $1, \Phi_{n}(\tau ; q)$ varies continuously from the initial guess $u_{n 0}(\tau)$ to the exact solution $u_{n}(\tau)$, so does $\Psi_{n}(q)$ from its initial frequency $\Omega_{n 0}$ to the nonlinear physical frequency $\Omega_{n}$. Similarly, $\Delta_{n}(q)$ varies from the initial approximation $\delta_{n 0}$ to the equilibrium position $\delta_{n}$.

By using the Taylor series expansion and considering the deformation derivatives, we will obtain

$$
\begin{gathered}
\Phi_{n}(\tau ; q)=u_{n 0}(\tau)+\sum_{m=1}^{+\infty} u_{n m}(\tau) q^{m}, \\
\Delta_{n}(q)=\delta_{n 0}+\sum_{m=1}^{+\infty} \delta_{n m} q^{m}, \quad \Psi_{n}(q)=\Omega_{n 0}+\sum_{m=1}^{+\infty} \Omega_{n m} q^{m},
\end{gathered}
$$

where

$$
\begin{gathered}
u_{n m}(\tau)=\left.\frac{1}{m !} \frac{\partial^{m} \Phi_{n}(\tau ; q)}{\partial q^{m}}\right|_{q=0}, \quad \delta_{n m}=\left.\frac{1}{m !} \frac{\partial^{m} \Delta_{n}(q)}{\partial q^{m}}\right|_{q=0}, \\
\Omega_{n m}=\left.\frac{1}{m !} \frac{\partial^{m} \Psi_{n}(q)}{\partial q^{m}}\right|_{q=0} .
\end{gathered}
$$

The $\hbar$ is an important auxiliary parameter that determines the convergence for the system. Furthermore, given that the auxiliary parameter $\hbar$ is properly chosen and all the series solutions are converging for $q=1$, the series solutions are written as

$$
\begin{gathered}
u_{n}(\tau)=u_{n 0}(\tau)+\sum_{m=1}^{+\infty} u_{n m}(\tau), \quad \delta_{n}=\delta_{n 0}+\sum_{m=1}^{+\infty} \delta_{n m}, \\
\Omega_{n}=\Omega_{n 0}+\sum_{m=1}^{+\infty} \Omega_{n m} .
\end{gathered}
$$

For the sake of brevity and simplicity, the following vectors are defined:

$$
\begin{gathered}
\mathbf{U}_{m}=\left\{u_{n 0}(\tau), u_{n 1}(\tau), \ldots, u_{n m}(\tau)\right\}, \\
\boldsymbol{\Delta}_{n m}=\left\{\delta_{n 0}, \delta_{n 1}, \ldots, \delta_{n m}\right\}, \\
\Psi_{n m}=\left\{\Omega_{n 0}, \Omega_{n 1}, \ldots, \Omega_{n m}\right\} .
\end{gathered}
$$

Differentiating the zeroth order deformation equation $m$ times with respect to the embedding parameters $q$, then dividing the resulting equations by $m$ ! and setting $q=0$, the $m$ th-order deformation equations are

$$
\mathscr{L}\left[u_{n m}(\tau)-\chi_{m} u_{n, m-1}(\tau)\right]=\hbar \mathbf{R}_{n m}\left(\mathbf{U}_{n, m-1}, \Delta_{n, m-1}, \Psi_{n, m-1}\right),
$$

which is subjected to the initial conditions

$$
u_{n m}(0)=0, \quad \dot{u}_{n m}(0)=0, \quad(m \geq 1),
$$

where

$$
\begin{gathered}
\chi_{m}= \begin{cases}0, & m \leq 1, \\
1, & m>1,\end{cases} \\
\mathbf{R}_{n m}=\left.\frac{1}{(m-1) !} \frac{\partial^{m-1} \mathcal{N}\left[\Phi_{n}(\tau ; q), \Delta_{n}(q), \Psi_{n}(q)\right]}{\partial q^{m-1}}\right|_{q=0} \\
=\sum_{k=0}^{m-1} \sum_{p=0}^{k} \Omega_{n p} \Omega_{n, k-p} \ddot{u}_{n, m-1-k}(\tau) \\
+\omega_{n}^{2}\left[u_{n, m-1}(\tau)+\delta_{n, m-1}\right] \\
+\Gamma_{n n n}\left\{\sum _ { k = 0 } ^ { m - 1 } \left[u_{n k}(\tau) u_{n, m-1-k}(\tau)+\delta_{n k} \delta_{n, m-1-k}\right.\right. \\
\left.\left.+2 u_{n k}(\tau) \delta_{n, m-1-k}\right]\right\} \\
+\Lambda_{n n n n}\left\{\sum_{k=0}^{m-1} \sum_{p=0}^{k} u_{n p}(\tau) u_{n, k-p}(\tau) u_{n, m-1-k}(\tau)\right. \\
+3 \Lambda_{n m n n}\left\{\sum_{k=0}^{m-1} \sum_{p=0}^{m} u_{n p}(\tau) u_{n, k-p}(\tau) \delta_{n, m-1-k} \sum_{p=0}^{k} \delta_{n p} \delta_{n, k-p} \delta_{n, m-1-k}\right\} \\
\left.+\sum_{k=0}^{m-1} \sum_{p=0}^{k} \delta_{n p} \delta_{n, k-p} u_{n, m-1-k}(\tau)\right\} .
\end{gathered}
$$

Moreover, the right hand side of the $m$ th-order deformation equation is expressed as

$$
\begin{aligned}
\mathbf{R}_{n m} & \left(\mathbf{U}_{n, m-1}, \boldsymbol{\Delta}_{n, m-1}, \boldsymbol{\Psi}_{n, m-1}\right) \\
= & c_{n, m 0}\left(\boldsymbol{\Delta}_{n, m-1}, \boldsymbol{\Psi}_{n, m-1}\right) \\
& +\sum_{k=1}^{\mu_{m}} c_{n, m k}\left(\boldsymbol{\Delta}_{n, m-1}, \boldsymbol{\Psi}_{n, m-1}\right) \cos (k \tau),
\end{aligned}
$$

where $c_{n, m 0}$ is the coefficient of the constant term, $c_{n, m k}$ is the coefficient of $\cos (k \tau)$, and $\mu_{m}$ is the positive integer dependent on order $m$. According to the property of the auxiliary linear operator $\mathscr{L}$, in order to avoid the constant 
drift term and the secular terms $\tau \cos \tau$, their coefficients are set to zero:

$$
\begin{array}{r}
c_{n, m 0}\left(\Delta_{n, m-1}, \Psi_{n, m-1}\right)=0, \quad c_{n, m 1}\left(\Delta_{n, m-1}, \Psi_{n, m-1}\right)=0, \\
(m=1,2,3, \ldots)
\end{array}
$$

which provide us with two additional algebraic equations for solving $\Omega_{n, m-1}$ and $\delta_{n, m-1}$. Consequently, given the unknown functions $\left(\omega_{n}^{2}, \Gamma_{n n n}, \Lambda_{n n n n}\right.$, and $\left.b_{n 0}\right)$, one can calculate the periodic solutions $u_{n m}(\tau)$ by solving the ordinary differential equation with the corresponding boundary conditions.

Therefore, the general periodic solution $u_{n m}(\tau)$ of (39) is obtained from

$$
\begin{aligned}
u_{n m}(\tau)= & \chi_{m} u_{n, m-1}(\tau)+C_{n 1} \sin \tau+C_{n 2} \cos \tau \\
& +\frac{\hbar}{\Omega_{n 0}^{2}} \sum_{k=2}^{\mu_{m}} \frac{c_{n, m k}\left(\Delta_{n, m-1}, \Psi_{n, m-1}\right)}{\left(1-k^{2}\right)} \cos (k \tau),
\end{aligned}
$$

where $C_{n 1}$ must be set to zero to obey the rule of solution expression and $C_{n 2}$ is a constant that could be determined by the initial conditions given by (40). Accordingly, the $m$ thorder analytic approximate solutions of $\delta_{n}, \Omega_{n}$, and $u_{n}(\tau)$ are

$$
\begin{gathered}
u_{n}(\tau) \approx u_{n 0}(\tau)+\sum_{m=1}^{m} u_{n m}(\tau), \quad \delta_{n} \approx \delta_{n 0}+\sum_{m=1}^{m} \delta_{n m} \\
\Omega_{n} \approx \Omega_{n 0}+\sum_{m=1}^{m} \Omega_{n m} .
\end{gathered}
$$

Here, we take $m=1$, for example, in order to illustrate the computational process of homotopy analysis method. In this case, the right hand side of the 1st order deformation equation could be expressed as

$$
\begin{aligned}
\mathbf{R}_{n 1}[ & \left.u_{n 0}(\tau), \delta_{n 0}, \Omega_{n 0}\right] \\
= & \Omega_{n 0}^{2} \ddot{u}_{n 0}(\tau)+\omega_{n}^{2}\left[u_{n 0}(\tau)+\delta_{n 0}\right]+\Gamma_{n n n}\left[u_{n 0}(\tau)+\delta_{n 0}\right]^{2} \\
& +\Lambda_{n n n n}\left[u_{n 0}(\tau)+\delta_{n 0}\right]^{3} \\
= & {\left[\omega_{n}^{2}+\frac{3}{4} b_{n 0}^{2} \Lambda_{n n n}+2 \Gamma_{n n n} \delta_{n 0}+3 \Lambda_{n n n n} \delta_{n 0}^{2}-\Omega_{n 0}^{2}\right] \cos (\tau) } \\
& +\left[\frac{1}{2} b_{n 0}^{2} \Gamma_{n n n}+\frac{2}{3} b_{n 0}^{2} \Lambda_{n n n n} \delta_{n 0}\right] \cos (2 \tau) \\
& +\left[\frac{1}{4} b_{n 0}^{3} \Lambda_{n n n n}\right] \cos (3 \tau) \\
& +\left(\frac{1}{2} b_{n 0}^{2} \Gamma_{n n n}+\omega_{n}^{2} \delta_{n 0}+\frac{3}{2} b_{n 0}^{2} \Lambda_{n n n n} \delta_{n 0}\right. \\
& \left.+\Gamma_{n n n} \delta_{n 0}^{2}+\Lambda_{n n n n} \delta_{n 0}^{3}\right) .
\end{aligned}
$$

In order to satisfy the rule of solution expression, the coefficients $c_{1,0}\left(\delta_{n 0}, \Omega_{n 0}\right)$ and $c_{1,1}\left(\delta_{n 0}, \Omega_{n 0}\right)$ must vanish. Therefore, we get two additional algebraic equations about $\Omega_{n 0}$ and $\delta_{n 0}$ :

$$
\begin{gathered}
\omega_{n}^{2}+\frac{3}{4} b_{n 0}^{2} \Lambda_{n m n n}+2 \Gamma_{n n n} \delta_{n 0}+3 \Lambda_{n n n n} \delta_{n 0}^{2}-\Omega_{n 0}^{2}=0 \\
\frac{1}{2} b_{n 0}^{2} \Gamma_{n n n}+\omega_{n}^{2} \delta_{n 0}+\frac{3}{2} b_{n 0}^{2} \Lambda_{n n n} \delta_{n 0}+\Gamma_{n n n} \delta_{n 0}^{2} \\
+\Lambda_{n n n n} \delta_{n 0}^{3}=0 .
\end{gathered}
$$

The solutions of (47) are

$$
\begin{gathered}
\Omega_{n 0}=\frac{1}{2} \sqrt{4 \omega_{n}^{2}+3 b_{n 0}^{2} \Lambda_{n n n n}+8 \Gamma_{n n n} \delta_{n 0}+12 \Lambda_{n n n n} \delta_{n 0}^{2}}, \\
\delta_{n 0}=-\frac{\Gamma_{n n n}}{3 \Lambda_{n n n n}}-\frac{\Upsilon_{n 1}}{3 \Lambda_{n n n n}} \sqrt[3]{\frac{\Upsilon_{n 2}+\sqrt{\Upsilon_{n 2}^{2}+32 \Upsilon_{n 1}^{3}}}{2}} \\
+\frac{1}{6 \Lambda_{n n n n}} \sqrt[3]{\frac{\Upsilon_{n 2}+\sqrt{\Upsilon_{n 2}^{2}+32 \Upsilon_{n 1}^{3}}}{2}}
\end{gathered}
$$

where

$$
\begin{gathered}
\Upsilon_{n 1}=-2 \Gamma_{n n n}^{2}+6 \omega_{n}^{2} \Lambda_{n n n n}+9 b_{n 0}^{2} \Lambda_{n n n n}^{2}, \\
\Upsilon_{n 2}=-16 \Gamma_{n n n}^{3}+72 \omega_{n}^{2} \Gamma_{n n n} \Lambda_{n n n n} .
\end{gathered}
$$

Eliminating the secular term and considering the expression of linear operator, the first order deformation equation becomes

$$
\begin{aligned}
\Omega_{n 0}^{2}\left[\ddot{u}_{n 1}(\tau)+u_{n 1}(\tau)\right] & \\
=\hbar & {\left[\left(\frac{1}{2} b_{n 0}^{2} \Gamma_{n n n}+\frac{2}{3} b_{n 0}^{2} \Lambda_{n n n n} \delta_{n 0}\right) \cos 2 \tau\right.} \\
& \left.+\left(\frac{1}{4} b_{n 0}^{3} \Lambda_{n n n n}\right) \cos 3 \tau\right] .
\end{aligned}
$$

It is easy to solve the linear ordinary differential equation with the initial conditions $\left(u_{n 1}(0)=0, \dot{u}_{n 1}(0)=0\right)$; therefore, the first order approximation is

$$
\begin{aligned}
u_{n 1}(\tau)= & \frac{\hbar}{96 \Omega_{n 0}^{2}} \\
& \times\left[\left(16 b_{n 0}^{2} \Gamma_{n n n}+3 b_{n 0}^{3} \Lambda_{n n n}+48 b_{n 0}^{2} \Lambda_{n n n n} \delta_{n 0}\right) \cos \tau\right. \\
& -\left(16 b_{n 0}^{2} \Gamma_{n n n}+48 b_{n 0}^{2} \Lambda_{n n n n} \delta_{n 0}\right) \cos 2 \tau \\
& \left.+\left(3 b_{n 0}^{3} \Lambda_{n n n n}\right) \cos 3 \tau\right] .
\end{aligned}
$$

Following the same procedure, the $m$ th-order $(m \geq 2)$ approximation of $\Omega_{n, m-1}, \delta_{n, m-1}$, and $u_{n m}(\tau)$ can be obtained. In general, the first order approximation of $q_{n}(t)$ obtained with the homotopy analysis method is expressed as follows:

$$
\begin{aligned}
q_{n}(t)= & u_{n 0}\left[\left(\Omega_{n 0}+\Omega_{n 1}\right) t\right]+u_{n 1}\left[\left(\Omega_{n 0}+\Omega_{n 1}\right) t\right] \\
& +\left(\delta_{n 0}+\delta_{n 1}\right) .
\end{aligned}
$$




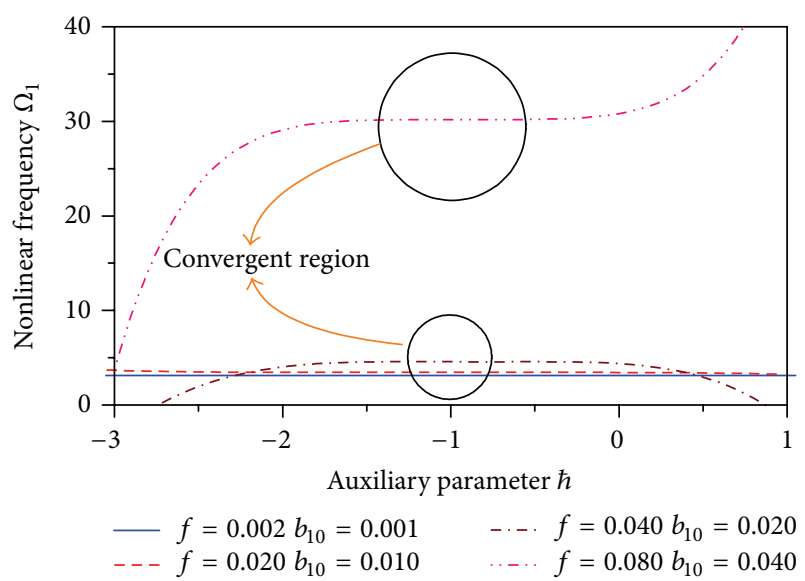

FIGURE 2: Effect of the auxiliary parameter $\hbar$ on the 1st symmetric mode frequency $\Omega_{1}$ obtained with the 5 th order homotopy analysis approximations for four different sag-to-span ratios $f$ and initial conditions $b_{10}$.

Finally, it should be pointed out that, on the one hand, only the zeroth order algebraic equations are nonlinear and all the higher order equations are linear. On the other hand, compared with the results obtained with Lindstedt-Poincare method ((19) and (20)), the zeroth order explicit expressions of the nonlinear frequency and displacements (48) are much more complex. Nevertheless, with the aid of the computer, the homotopy analysis method still provides us with a very convenient way to obtain the higher order approximations.

\section{Numerical Results and Discussions}

The dimensional parameters and material properties of the suspended cable are chosen as follows [24]: the area of the cross-section $A=0.1257 \mathrm{~mm}^{2}$, the mass per unit length $m=$ $4.8655 \times 10^{-5} \mathrm{~kg} / \mathrm{m}$, the Young modulus $E=1340.83 \mathrm{MPa}$, and the cable span $l=600.5 \mathrm{~mm}$. Moreover, four different sag-to-span ratios $(f=0.002,0.02,0.04$, and 0.08 ) are chosen to study the differences between these two analytical approaches in the case of the nonlinear free vibrations of suspended cables.

4.1. Convergence and Accuracy of Solutions. As mentioned in the previous section, the auxiliary parameter $\hbar$ plays an important role in the convergence for the approximate series solutions obtained with the homotopy analysis method. Figure 2 shows the effect of the auxiliary parameter $\hbar$ on the 5 th order series solutions for the 1st symmetric mode frequency $\Omega_{1}$. In order to make the research less complex, as to every sag-to-span ratio $f$, only one initial condition is selected $\left(b_{10}=f / 2\right)$. As indicated in Figure 2, there is a convergent region $(\hbar \in[-2.0,0])$ for the 5 th order approximations. Therefore, the auxiliary parameter $\hbar$ is chosen as -1.0 in the following study.

Nevertheless, it should be mentioned that the convergence tests or proofs are significant and important for homotopy analysis method. Yet, only several sag-to-span ratios

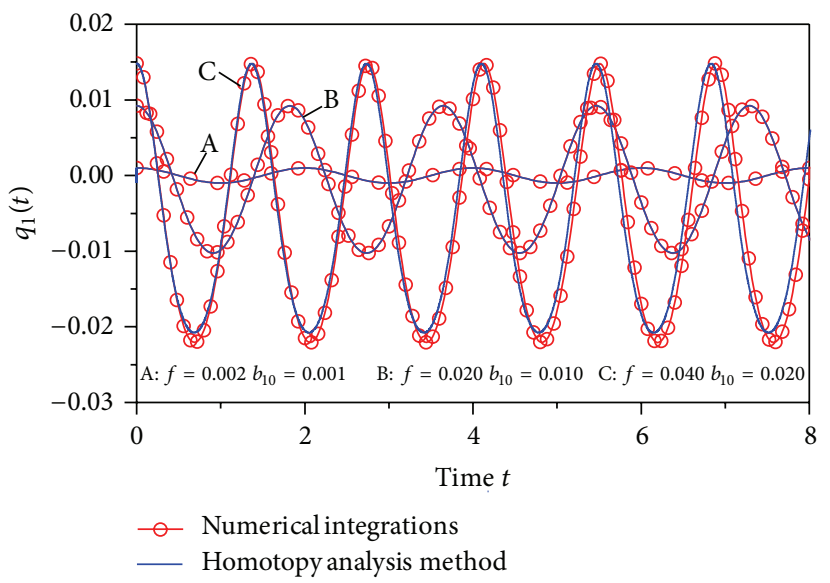

FIgURE 3: Comparison of the series solution $q_{1}(t)$ obtained with the 1 st order homotopy analysis method and numerical integrations in the case of the 1st symmetric mode.

and initial conditions are involved, and the convergent regions could not be checked one by one. Moreover, just as mentioned by Liao [13], it deserves to be further studied in which the auxiliary parameter $\hbar$ and function $H(\tau)$ for any given nonlinear problem should be chosen. Therefore, the homotopy analysis method needs further improvement and development in this respect.

Once the auxiliary parameter $\hbar$ is chosen appropriately, approximate series solutions in the case of nonlinear free vibrations could be obtained. Moreover, in order to verify the approximations obtained with the analytical approaches, we could substitute the initial condition $b_{n 0}$ and the equilibrium position $\delta_{n}$ into the initial conditions (22), and then the numerical integrations are applied to obtain the exact solutions. It is time consuming to obtain the results with regular numerical methods for the undamped periodic free oscillations, though. The comparison of the series solution $q_{1}(t)$ obtained with the homotopy analysis method and numerical integrations in the case of nonlinear free vibration with the 1st symmetric mode is made in Figure 3. It is noted that the first order approximations obtained with the homotopy analysis method are in good agreement with the exact ones obtained through numerical integrations in these three different cases.

4.2. Frequency Amplitude Relationships. Generally speaking, both the frequency amplitude relationship and the effect of the nonlinearities on the law of motion are two important aspects that need to be examined and analyzed [4]. Furthermore, owing to large flexibility, light weight, and low inherent damping of the suspended cable, this system is often susceptible to exhibit large amplitude vibrations. Therefore, in the following, both of these two aspects are investigated and illustrated by using the homotopy analysis method and Lindstedt-Poincare method. In addition, in order to clarify the validity of the obtained results, some numerical results of the original ordinary differential equation (ODE) are given. 
TABLE 1: Three nondimensional parameters of suspended cables.

\begin{tabular}{lcccc}
\hline & $(\mathrm{a})$ & $(\mathrm{b})$ & $(\mathrm{c})$ & $(\mathrm{d})$ \\
\hline$f$ & 0.002 & 0.02 & 0.04 & 0.08 \\
$\alpha$ & 9.418 & 94.181 & 188.361 & 376.723 \\
$\lambda^{2}(\lambda / \pi)$ & $0.0024(0.0156)$ & $2.4110(0.4945)$ & $19.2882(1.3987)$ & $154.3060(3.9560)$ \\
\hline
\end{tabular}

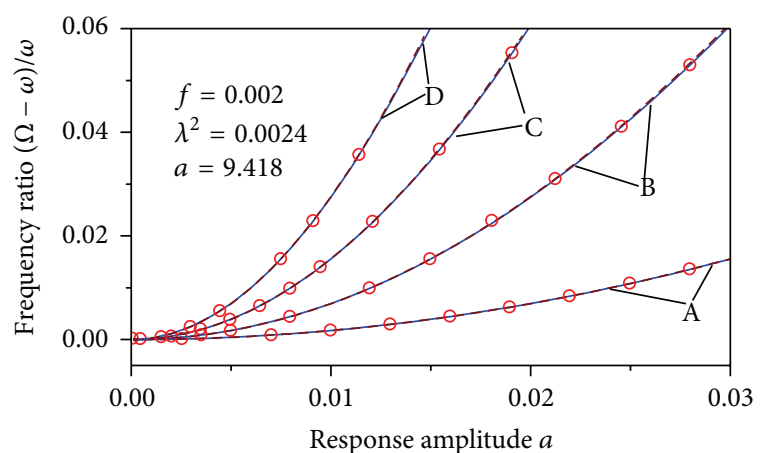

(a)

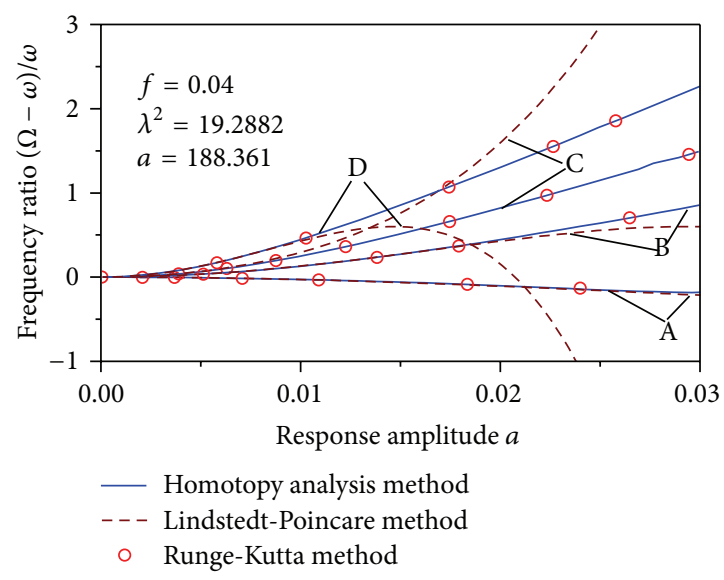

(c)

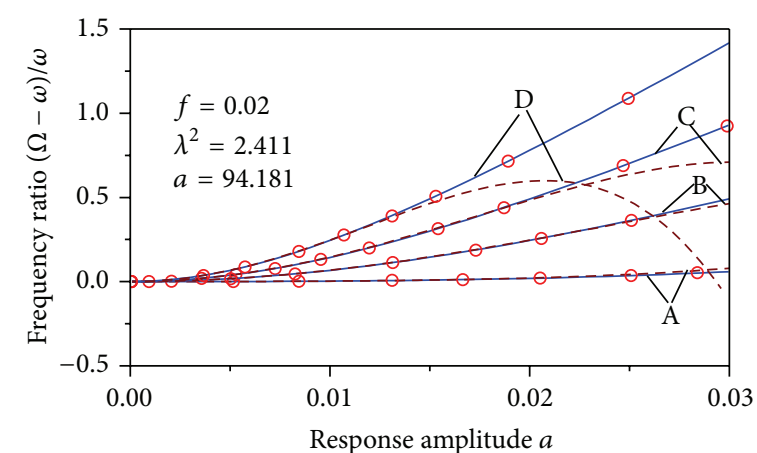

(b)

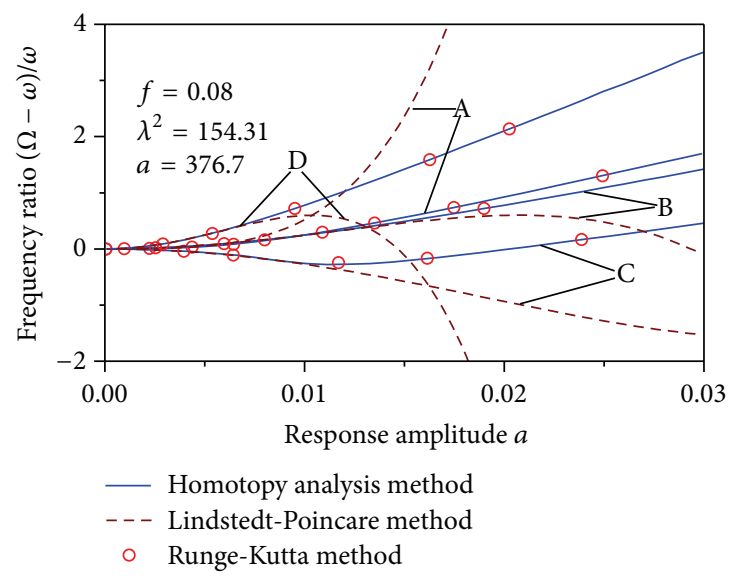

(d)

Figure 4: Comparisons of frequency amplitude relationships obtained with the 1st order homotopy analysis method and the 4th order Lindstedt-Poincare method for the first four modes: (a) $f=0.002$; (b) $f=0.02$; (c) $f=0.04$; (d) $f=0.08$. A: the 1st symmetric mode, B: the 1st antisymmetric mode, C: the 2 nd symmetric mode, and $\mathrm{D}$ : the 2 nd antisymmetric mode.

At the beginning, Table 1 illustrates the following three nondimensional parameters: the sag-to-span ratio $f$, the Irvine parameter $\lambda^{2}$, and the nondimensional parameter $\alpha(E A / H)$. As is shown in Table 1 , with the rise of the sag-tospan ratio $f$, the Irvine parameter $\lambda^{2}$ increases very quickly and the coefficients of the quadratic and cubic nonlinearities become very large, too. Therefore, in the case of the large sagto-span ratios, there is no small parameter in the equation of motion. On the other hand, it should be noticed that the hardening or softening characteristic of the suspended cable is largely dependent on the predominance of either the quadratic or the cubic nonlinearity term.

Figure 4 shows the frequency amplitude relationships of suspended cables under four different sag-to-span ratios ( $f=$ $0.002,0.02,0.04$ and 0.08 ) for the first two symmetric and antisymmetric modes. Moreover, the nondimensionalization of cable amplitude with respect to cable span is considered (4), and this matter affects the range of the response amplitude. In fact, as to the suspended cable, no matter the sag-to-span ratio is large or small, it is often susceptible to exhibit large response amplitude vibration, so the range of the dimensional response amplitude is chosen as $[0,0.03]$ for different sag-to-span ratios and vibration mode shapes. Nevertheless, it should be explained that, as to higher order vibration mode shapes, too large amplitude vibrations may not be easy to exhibit.

In Figure 4(a), we show the frequency amplitude relationships of the suspended cable when the sag-to-span ratio $f=0.002$. In this case, the suspended cable corresponds to a taut-string, and the contribution of the cubic nonlinearity term is dominant in the nonlinear responses. Therefore, as shown in Figure 4(a), the suspended cable exhibits only 
hardening behavior for both the symmetric modes (A and C) and antisymmetric modes (B and D). Moreover, the frequency amplitude relationships do not exhibit quantitative and qualitative differences in the whole range of the response amplitude, and excellent agreements between the homotopy analysis method and Lindstedt-Poincare method for the first four modes are presented in Figure 4(a). The numerical integrations show that both of these two analytical methods are appropriate in the case of the taut-string no matter the response amplitude is large or small.

As the sag-to-span ratio increases $(f=0.02)$ in Figure 4(b), the contribution of the quadratic nonlinearity term is still small when compared with that of the cubic one. Therefore, the suspended cable still exhibits only hardening behavior. Furthermore, according to the conclusions obtained by Rega et al. [8], the dynamic behavior of the suspended cable when the sag-to-span ratio $f=0.02$ is strictly hardening. Nevertheless, as described in Figure 4(b), although for the 1st symmetric and antisymmetric modes (A and $\mathrm{B}$ ), there are not too many quantitative differences between the results obtained with these two approaches, whereas for the 2nd symmetric and antisymmetric modes $(\mathrm{C}$ and $\mathrm{D})$, the differences in the curves obtained by using these two analytical approaches increase with the rise of the vibration amplitude. To be more specific, the curves obtained with the Lindstedt-Poincare method start hardening, and they are in good agreement with the curves obtained with the homotopy analysis method. However, as the response amplitude increases, the curves obtained with LindstedtPoincare method then become softening. Hence, provided that the response amplitude of the suspended cable is large, the Lindstedt-Poincare method fails to reflect the characteristic of the suspended cable appropriately. However, as one of the typical perturbation methods, too large amplitude values are likely considered for the Lindstedt-Poincare solutions here, which is known to hold only for small nonlinearities. Furthermore, as the vibration mode increases, the mode shapes become more constrained and the agreement for the first mode (A and B) is good up to 0.025 , while the one for the second modes (C and D) occurs up to considerably lower amplitude values, with also a further decrease when passing from the 2 nd symmetric $(\mathrm{C})$ to the 2 nd antisymmetric mode (D).

Figure 4(c) describes the frequency amplitude relationships of the suspended cable when the sag-to-span ratio $f=0.04$ and excellent agreements between the solutions of the homotopy analysis method and numerical integrations are presented, both for the symmetric modes and antisymmetric modes. However, compared with the LindstedtPoincare method, there are some qualitative and quantitative differences between the analytical results. Firstly, the dynamic behavior of the suspended cable is initially softening at a low value of the response amplitude of the 1st symmetric mode (A), but, at a higher value of it, because the cubic nonlinearity term may dominate the nonlinear free oscillations, the dynamic behavior becomes hardening again. Provided that the suspended cable vibrates at low values of the response amplitude (e.g., $a \leq 0.03$ ), the differences between these two methods could be neglected, whereas if the response amplitude of the suspended cable is large, there are quantitative differences between them. Secondly, for the 2nd symmetric mode (C) when the sag-to-span ratio $f=0.04$, although the general trend of frequency amplitude relationship obtained with these two analytical approaches makes no great differences, with the increase of the response amplitude, the Lindstedt-Poincare method predicts more hardening behavior than the homotopy analysis method does. Thirdly, for the 1st and 2nd antisymmetric modes (B and $\mathrm{D})$, the coefficient of the quadratic nonlinearity term equals to zero $\left(\Gamma_{n n n} \equiv 0\right)$. Therefore, the dynamic behavior of the suspended cable is definitely hardening in the whole range of the vibration amplitude for any antisymmetric modes. As described in Figure 4(c), both of the frequency amplitude relationships exhibit hardening behavior in the case of the small amplitude vibration. Nevertheless, as the response amplitude increases, the dynamic characteristic of the suspended cable obtained with the Lindstedt-Poincare method becomes softening. Therefore, as to the antisymmetric mode $(f=0.04)$, the scope of the response amplitude should be considered with care, provided that the dynamic behavior of the suspended cable needs to be reflected accurately by using the Lindstedt-Poincare method. Furthermore, by comparing the lower order modes ( $\mathrm{A}$ and $\mathrm{C}$ ) with the higher order ones (B and D), the accuracy of the solutions obtained with the Lindstedt-Poincare decreases with the increase of the mode order, due to the more constrained mode shapes.

Furthermore, Figure 4(d) displays the frequency amplitude relationships of the suspended cable for the first two symmetric and antisymmetric modes when the sag-to-span ratio increases continually to 0.08 . In this case, for the 1st symmetric mode (A), the effect of the cubic nonlinearity term plays a dominant role in the nonlinear vibration and the characteristic of the results obtained with the homotopy analysis method is hardening, whereas the LindstedtPoincare method predicts more hardening behavior. Besides, as to the 2nd symmetric mode (C), the suspended cable exhibits hardening or softening characteristic behavior which is dependent on the vibration amplitude. Although the general trend of the frequency amplitude relationship obtained with these two methods shows no great difference, the significant quantitative difference could be observed for the 2nd symmetric mode. What is more, as to the 1st and 2nd antisymmetric modes, just as mentioned in the previous analysis, if the dynamic behavior of the suspended cable obtained with the Lindstedt-Poincare method needs to be reflected correctly, the range of the response amplitude is limited, especially for the higher order modes. As illustrated in Figure 4(d), it is found that the range of agreement is now larger for the first antisymmetric mode (B) than for the symmetric one (A), because after the first crossover $(\lambda / \pi>$ 2.0) the former is the one with the less constrained mode shape while the latter exhibits now three half waves which is described in Figure 5(d).

4.3. Displacement Fields. In the following, a comparison of displacement fields obtained with these two analytical approaches is made. Substituting (7), (20), and (52) into (10), the expressions of the displacement field $v(x, t)$ are 


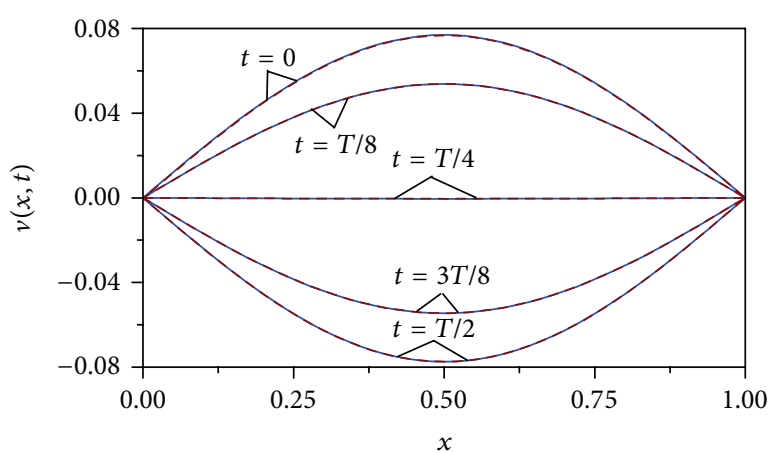

(a)

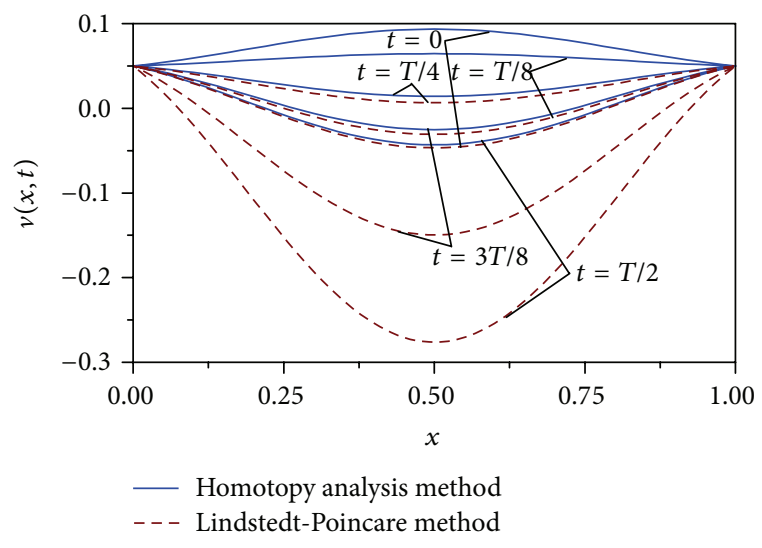

(c)

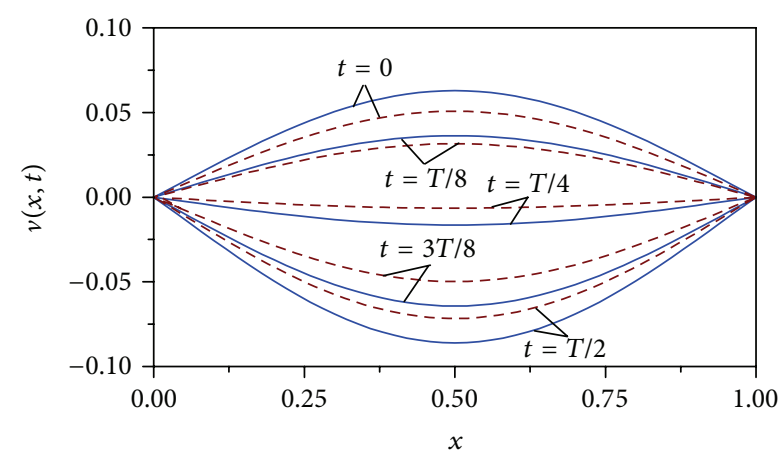

(b)

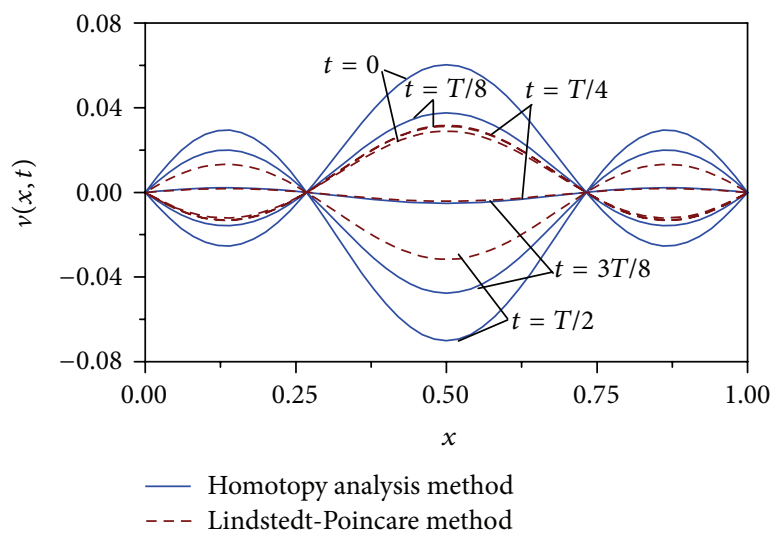

(d)

FIGURE 5: In-plane displacement fields obtained with homotopy analysis method and Lindstedt-Poincare method in the case of the first symmetric mode: (a) $f=0.002,\left(\Omega_{1}-\omega_{1}\right) / \omega_{1}=0.05$; (b) $f=0.02,\left(\Omega_{1}-\omega_{1}\right) / \omega_{1}=0.25$; (c) $f=0.04,\left(\Omega_{1}-\omega\right) / \omega_{1}=-0.10$; (d) $f=0.08$, $\left(\Omega_{1}-\omega_{1}\right) / \omega_{1}=2.0$.

obtained. Figure 5 shows the in-plane displacement fields obtained with the homotopy analysis method and LindstedtPoincare method in the case of the 1st symmetric mode. For the sake of convenience, only four displacement fields are chosen and illustrated. As could be observed in Figure 5(a), the solutions obtained with Lindstedt-Poincare method are in good agreement with the ones obtained with homotopy analysis method when $f=0.002$ and $\left(\Omega_{1}-\omega_{1}\right) / \omega_{1}=0.05$. Nevertheless, in some other cases, quantitative differences could be observed (Figures 5(b), 5(c), and 5(d)) and the location of the maximum amplitude varies with the change of the space and time.

It should be mentioned that, as to the first three cases, the Irvine parameter $\lambda / \pi<2$ and the first symmetric mode frequency is less than the first antisymmetric one. Nevertheless, in the last case in our study, the Irvine parameter $\lambda / \pi$ is chosen as $3.9560(\lambda / \pi>2)$. As shown in Figure 5(d), the frequency of the first symmetric mode is larger than the one of the first antisymmetric mode and the vertical component of the first symmetric mode has two internal nodes and exhibits now three half waves.

4.4. Axial Tension Forces. In the fields of engineering, the tension force of the suspended cable plays a very important role. Therefore, the cable total tension obtained with different analytic methods is studied and analyzed in this section. According to Srinil et al. [10], the nondimensional cable total tension is expressed as

$$
\begin{aligned}
H_{T}(t)= & 1+\alpha q_{n}(t) \int_{0}^{1} y^{\prime}(x) \varphi_{n}^{\prime}(x) \mathrm{d} x \\
& +\frac{\alpha}{2} q_{n}^{2}(t) \int_{0}^{1} \varphi_{n}^{\prime 2}(x) \mathrm{d} x,
\end{aligned}
$$

where the quasi-static assumption is applied. It is interesting to find out that the nondimensional cable total tension is independent of the coordinate $x$.

Figure 6 describes the time histories of the nondimensional cable tension force in the case of the first symmetric mode, and no negative horizontal tension force is observed. A good qualitative or quantitative agreement of the nondimensional cable total tension is presented in Figure 6(a). Figure 6(b) displays that the general trends of the time histories obtained with different methods are the same, except the peak value of the cable tension force which is very important in suspended cables designing to ensure sufficient security coefficient. Moreover, it is noted that in Figure 6(c) the tiny difference in the vibration amplitude may lead to significant quantitative differences in cable total 


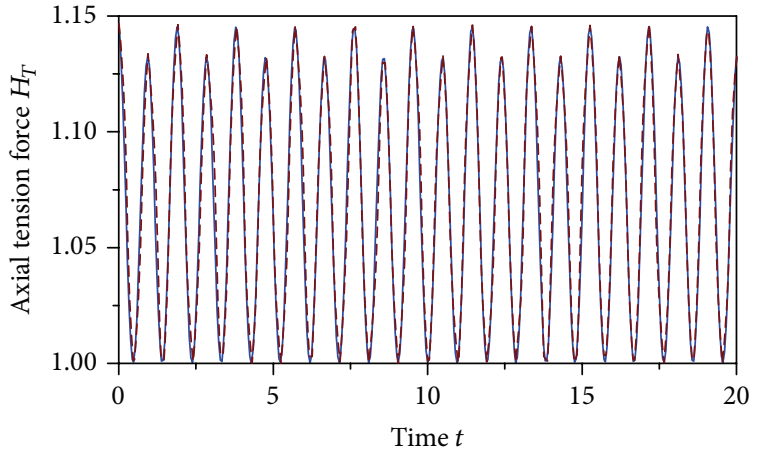

(a)

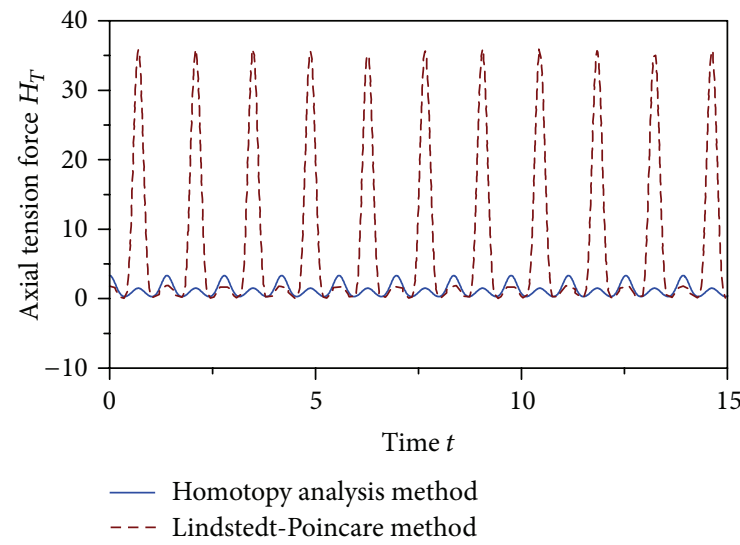

(c)

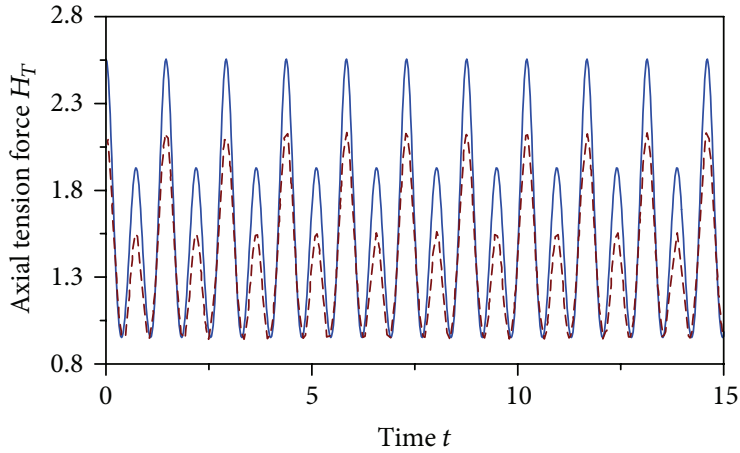

(b)

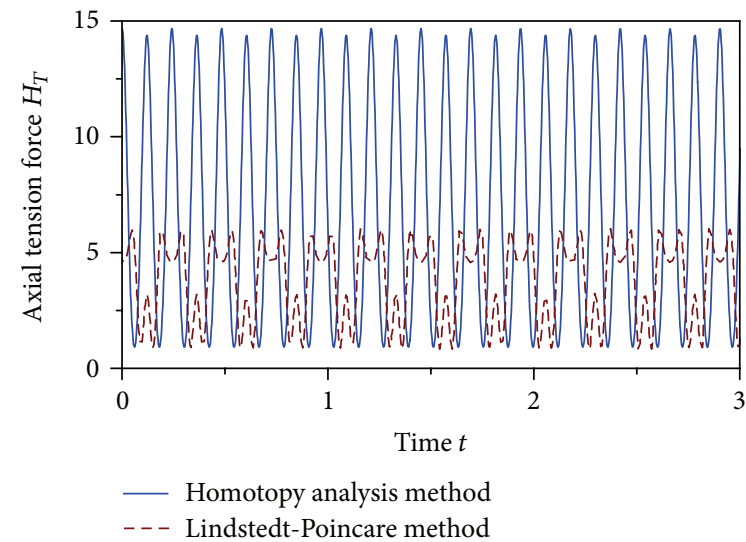

(d)

FiguRE 6: Time histories of the nondimensional cable tension force for the case of the first symmetric mode: (a) $f=0.002,\left(\Omega_{1}-\omega_{1}\right) / \omega_{1}=0.05$; (b) $f=0.02,\left(\Omega_{1}-\omega_{1}\right) / \omega_{1}=0.25$; (c) $f=0.04,\left(\Omega_{1}-\omega_{1}\right) / \omega_{1}=-0.10$; (d) $f=0.08,\left(\Omega_{1}-\omega_{1}\right) / \omega_{1}=2.0$.

tension. Finally, in Figure 6(d), one of the evident differences is the time of the minimum value of the cable total tension.

\section{Conclusions}

In this research, the nonlinear free vibrations of the singlemode model of the suspended cable are studied via the Lindstedt-Poincare method, homotopy analysis method, and numerical integrations and only the first two symmetric and antisymmetric modes are considered. Moreover, the numerical results and discussions are extended from a taut string $(f=0.002)$ to a slack cable $(f=0.08)$.

The homotopy analysis method does not depend on any small parameter assumption and provides us with a convenient way to ensure the convergence for the series solutions. It is found that above a certain value of response amplitude, it still continues to agree well with the results of the numerical integrations of the ODEs, whereas the Lindstedt-Poincare solutions partly fail. On the one hand, in the case of the taut string (small sag-to-span ratio), these two analytical methods make no difference. On the other hand, as to the high order vibration modes, large response amplitudes, and sag-to-span ratios, these two approaches may lead to some quantitative and qualitative differences in the frequency amplitude relationships. However, the results obtained with the homotopy analysis method are in good agreement with the ones obtained by using numerical integrations in the whole range of response amplitude. Furthermore, the homotopy analysis method has an advantage over the original one in the accuracy of the estimation of the displacement fields and second order harmonic to the time history of the cable axial tension.

\section{Conflict of Interests}

The authors declare that there is no conflict of interests regarding the publication of this paper.

\section{Acknowledgments}

The work was supported by the National Natural Science Foundation of China (nos. 11032004 and 11102063). The authors would like to thank the anonymous reviewers for their constructive comments and suggestions on the earlier version of this paper.

\section{References}

[1] H. M. Irvine, Cable Structures, The MIT Press, Cambridge, Mass, USA, 1981. 
[2] S. W. Rienstra, "Nonlinear free vibrations of coupled spans of overhead transmission lines," Journal of Engineering Mathematics, vol. 53, no. 3-4, pp. 337-348, 2005.

[3] G. Rega, "Nonlinear vibrations of suspended cables. Part I: modeling and analysis," Applied Mechanics Reviews, vol. 57, no. 1-6, pp. 443-478, 2004.

[4] G. Rega, "Nonlinear vibrations of suspended cables. Part II: deterministic phenomena," Applied Mechanics Reviews, vol. 57, no. 1-6, pp. 479-514, 2004.

[5] P. Hagedorn and B. Schäfer, "On non-linear free vibrations of an elastic cable," International Journal of Non-Linear Mechanics, vol. 15, no. 4-5, pp. 333-340, 1980.

[6] A. Luongo, G. Rega, and F. Vestroni, "Monofrequent oscillations of a non-linear model of a suspended cable," Journal of Sound and Vibration, vol. 82, no. 2, pp. 247-259, 1982.

[7] A. Luongo, G. Rega, and F. Vestroni, "Planar non-linear free vibrations of an elastic cable," International Journal of NonLinear Mechanics, vol. 19, no. 1, pp. 39-52, 1984.

[8] G. Rega, F. Vestroni, and F. Benedettini, "Parametric analysis of large amplitude free vibrations of a suspended cable," International Journal of Solids and Structures, vol. 20, no. 2, pp. 95-105, 1984.

[9] F. Benedettini, G. Rega, and F. Vestroni, "Modal coupling in the free nonplanar finite motion of an elastic cable," Meccanica, vol. 21, no. 1, pp. 38-46, 1986.

[10] N. Srinil, G. Rega, and S. Chucheepsakul, "Three-dimensional non-linear coupling and dynamic tension in the largeamplitude free vibrations of arbitrarily sagged cables," Journal of Sound and Vibration, vol. 269, no. 3-5, pp. 823-852, 2004.

[11] K. Takahashi and Y. Konishi, "Non-linear vibrations of cables in three dimensions. Part I: non-linear free vibrations," Journal of Sound and Vibration, vol. 118, no. 1, pp. 69-84, 1987.

[12] S. J. Liao, The proposed homotopy analysis techniques for the solution of nonlinear problems [Ph.D. thesis], Shanghai Jiao Tong University, 1992.

[13] S. J. Liao, Beyond Perturbation: Introduction to the homotopy Analysis Method, Chapman and Hall/CRC Press, Boca Raton, Fla, USA, 2003.

[14] S. H. Hoseini, T. Pirbodaghi, M. Asghari, G. H. Farrahi, and M. T. Ahmadian, "Nonlinear free vibration of conservative oscillators with inertia and static type cubic nonlinearities using homotopy analysis method," Journal of Sound and Vibration, vol. 316, no. 1-5, pp. 263-273, 2008.

[15] T. Pirbodaghi, M. T. Ahmadian, and M. Fesanghary, "On the homotopy analysis method for non-linear vibration of beams," Mechanics Research Communications, vol. 36, no. 2, pp. 143-148, 2009.

[16] M. H. Kargarnovin and R. A. Jafari-Talookolaei, "Application of the homotopy method for the analytic approach of the nonlinear free vibration analysis of the simple end beams using four engineering theories," Acta Mechanica, vol. 212, no. 3-4, pp. 199-213, 2010.

[17] Y. H. Qian, D. X. Ren, S. K. Lai, and S. M. Chen, "Analytical approximations to nonlinear vibration of an electrostatically actuated microbeam," Communications in Nonlinear Science and Numerical Simulation, vol. 17, no. 4, pp. 1947-1955, 2012.

[18] Y. H. Qian, W. Zhang, B. W. Lin, and S. K. Lai, "Analytical approximate periodic solutions for two-degree-of-freedom coupled van der Pol-Duffing oscillators by extended homotopy analysis method," Acta Mechanica, vol. 219, no. 1-2, pp. 1-14, 2011.
[19] R. Wu, J. Wang, J. Du, Y. Hu, and H. Hu, "Solutions of nonlinear thickness-shear vibrations of an infinite isotropic plate with the homotopy analysis method," Numerical Algorithms, vol. 59, no. 2, pp. 213-226, 2012.

[20] P.-X. Yuan and Y.-Q. Li, "Primary resonance of multiple degreeof-freedom dynamic systems with strong non-linearity using the homotopy analysis method," Applied Mathematics and Mechanics, vol. 31, no. 10, pp. 1293-1304, 2010.

[21] Y. Tan and S. Abbasbandy, "Homotopy analysis method for quadratic Riccati differential equation," Communications in Nonlinear Science and Numerical Simulation, vol. 13, no. 3, pp. 539-546, 2008.

[22] X. You and H. Xu, "Analytical approximations for the periodic motion of the Duffing system with delayed feedback," Numerical Algorithms, vol. 56, no. 4, pp. 561-576, 2011.

[23] Y. Y. Zhao, L. H. Wang, W. C. Liu, and H. B. Zhou, "Direct treatment and discretization of nonlinear dynamics of suspended cable," Acta Mechanica Sinica, vol. 37, pp. 329-338, 2005 (Chinese).

[24] G. Rega, W. Lacarbonara, A. H. Nayfeh, and C. M. Chin, "Multiple resonances in suspended cables: direct versus reduced-order models," International Journal of Non-Linear Mechanics, vol. 34, no. 5, pp. 901-924, 1999. 

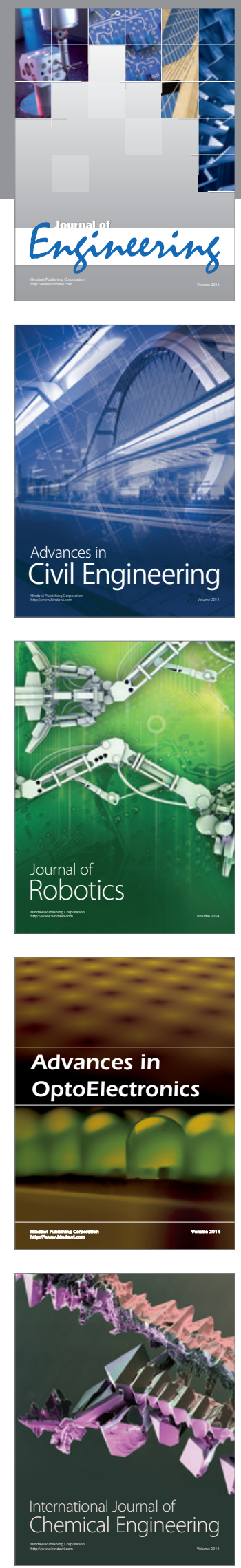

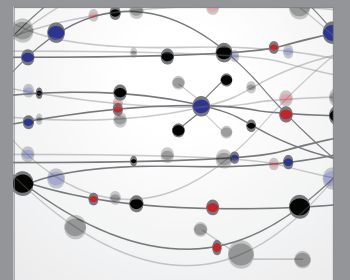

The Scientific World Journal
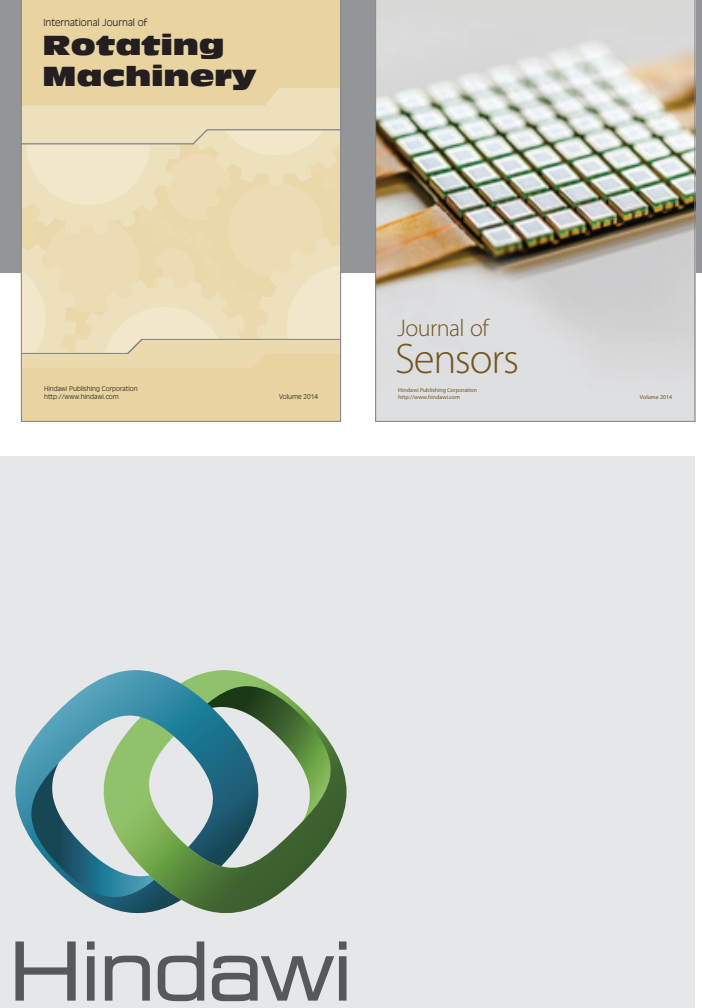

Submit your manuscripts at http://www.hindawi.com
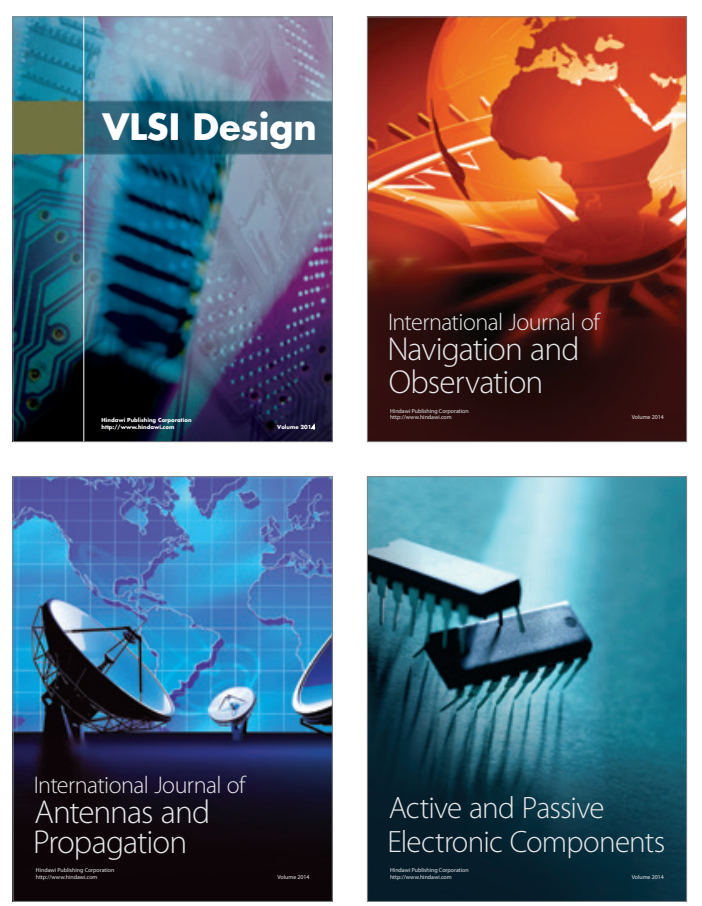
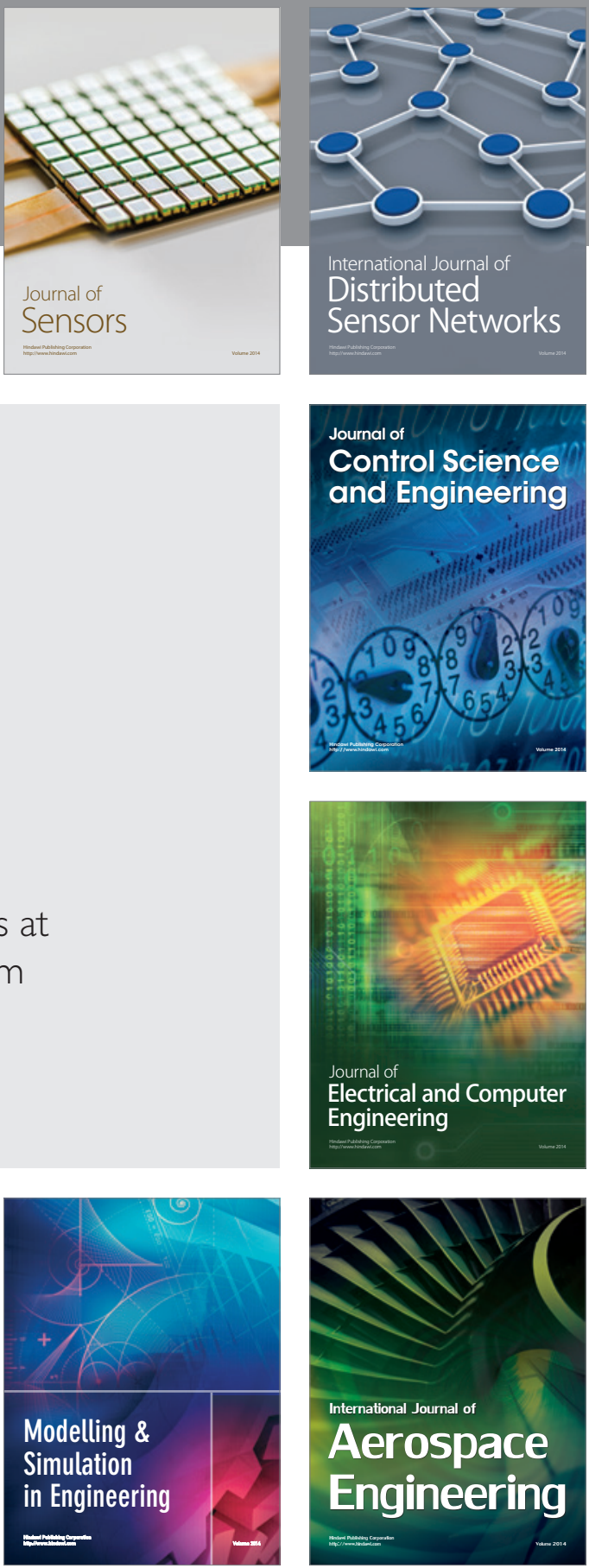

Journal of

Control Science

and Engineering
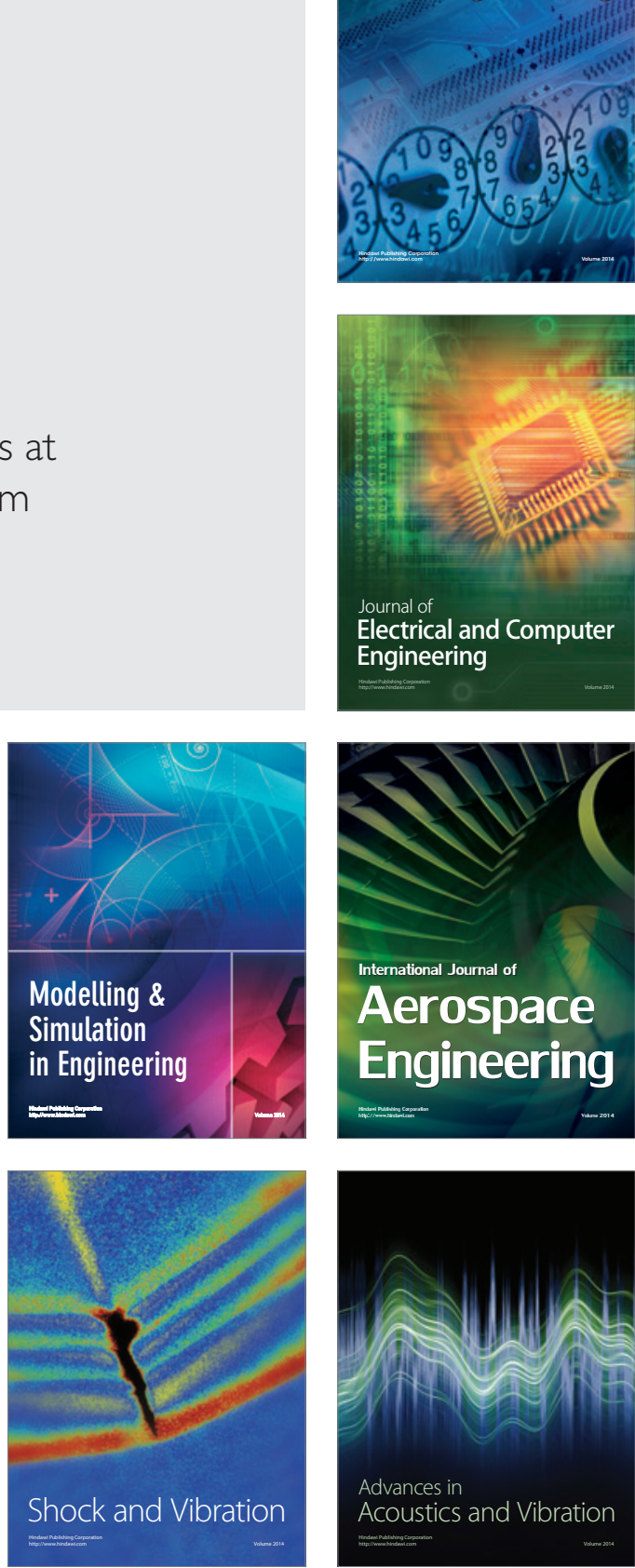